\title{
Solução IoT de Monitoramento de Poços para Gerenciamento de Recursos Hídricos
}

\author{
Estudo de caso para aplicado na região metropolitana do Recife
}

\author{
Victor Mendonça de Azevedo ${ }^{1}$ (D) orcid.org/0000-0003-2943-4622 \\ Alexandre Magno Andrade Maciel 1 (D) orcid.org/0000-0003-4348-9291 \\ Kiev Santos da Gama 2 (D) orcid.org/0000-0003-1508-6196 \\ ${ }^{1}$ Escola Politécnica de Pernambuco, Universidade de Pernambuco, Recife, Brasil,
${ }^{2}$ Centro de Informática, Universidade Federal de Pernambuco, Recife, Pernambuco, Brasil,
E-mail do autor principal: vma@ecomp.poli.br
}

\section{Resumo}

O objetivo principal das cidades inteligentes é alcançar o uso sustentável dos recursos. Para fazer o uso correto dos recursos, é necessário um monitoramento e gerenciamento precisos. Em alguns lugares, como os aquíferos subterrâneos, o acesso para monitoramento pode ser difícil, portanto, o uso de sensores pode ser uma boa solução. Os aquíferos subterrâneos são um importante recurso hídrico. O correto monitoramento, ajuda a prevenir seu esgotamento e a evitar impactos ambientais. Este artigo tem como objetivo apresentar um esforço que vem sendo realizado pela Agência Pernambucana de Águas e Clima - APAC, no monitoramento de poços subterrâneos, com o uso de uma solução IoT para um monitoramento continuo e em tempo real, com a análise dos dados obtidos e geração de histórico, possibilitando o estudo do comportamento dos aquíferos subterrâneos da Região Metropolitana de Recife. Apresentamos como essa solução é composta e como os dados gerados podem ser analisados.

Palavras-Chave: Aquíferos; Condutividade; IoT; Análise de dados; Poços;

\section{Abstract}

The main goal of smart cities is to achieve the sustainable use of resources. Accurate monitoring and management is required to make the correct use of resources. In some places, such as underground aquifers, access for monitoring can be difficult, so the use of sensors can be a good solution. Underground aquifers are an important water resource. Correct monitoring helps prevent depletion and avoid environmental impacts. This paper aims to present an effort that has been carried out by the Pernambuco Water and Climate Agency (APAC), in the monitoring of underground wells, with the use of an IoT solution for a continuous and real time monitoring, with the analysis of the obtained data and generation of history, it could enable the study of the behavior of subterranean aquifers of the Metropolitan Region of Recife. We present how this solution is composed and how the generated data can be analyzed.

Key-words: Aquifers; Conductivity; IoT; Data Analysis; Wells 


\section{Introdução}

O surgimento de novas tendências sempre vem acompanhado de tecnologias de base existentes, que fornecem os meios para sua popularização. Este também é o caso da IoT (Internet of Things, ou Internet das Coisas) e sua relação com o que é chamado de Big Data. Enquanto a IoT conecta diversos dispositivos e sistemas por meio de sensores (relação máquinamáquina), tornando cada vez menor a necessidade de interação humana nestes processos, o Big Data toma conta da imensa quantidade de dados gerados.

Em uma pesquisa realizada pela Gartner em 2014, analistas previram que em 2020 a quantidade de dispositivos conectados passará dos atuais 5 bilhões para 25 bilhões [1].

A IoT vem para facilitar ainda mais, tornando os dispositivos móveis conectados à internet verdadeiros chamarizes de informação e produtores de dados. Só que essa grande quantidade de dados deve ir para algum lugar para não se perder, e deve ainda ser "minerada" para ter valor agregado e ser passível de utilização. Neste cenário entra a Big Data.

O termo Big Data é dado ao armazenamento e processamento da mistura de dados estruturados (números) e não-estruturados (imagem, mídias sociais, Twitter, etc.). Trabalha com grande volume de dados em uma velocidade de aquisição que pode até ser em tempo real, ao mesmo tempo em que recebe diferentes tipos de entradas.

Através do uso de técnicas de análise estatística automatizada, ou seja, o Data Mining, as empresas estão descobrindo as tendências e padrões de comportamento que antes passava despercebidos. Uma vez descoberta essa inteligência vital, ela pode ser usada de forma preditiva para uma variedade de coisas.

O primeiro passo de para construir um programa de Mineração de Dados é a Coleta de Dados. A maioria das empresas já realizam essas tarefas de coleta de dados, contudo, nem todos esses dados coletados são valiosos, sendo necessária uma seleção.

A chave aqui é para localizar os dados críticos para o negócio, refiná-lo e prepará-lo para o processo de Mineração de Dados. Dados úteis não são mais chamados de dados, mas sim de 13 informação. Dessa maneira, informações já possuem aspectos úteis e valor agregado.

A mineração de dados busca encontrar padrões (classificar e criar modelos de predição), segmentar informações (isolando variáveis) ou buscar correlações entre dados existentes. É aí que reside a grande vantagem da Big Data. Estes métodos de coleta, armazenamento e pósprocessamento que são utilizados em conjunto com a IoT.

Os sistemas de sensores e monitoramentos estão fornecendo atualmente às empresas de serviços de água grandes quantidades de fluxos de dados em tempo real. No entanto, esse fluxo de dados ainda não é chamado de Big Data. Big Data abrange muito mais. Para exemplificar esse fato, considere os dados derivados de sensores instalados em bombas de água, que são vistos em monitores em qualquer momento, mas os dados desses sistemas de supervisão e aquisição de dados (SCADA) se concentram no que está acontecendo agora ou no que aconteceu no passado. Esta situação seria como olhar em um espelho retrovisor de um carro, não se torna muito eficaz se o caminho tiver muitas curvas a frente. Felizmente, esses sistemas SCADA poderiam potencialmente dizer muito mais, poderiam melhorar a eficiência, o uso de energia e as condições da rede que podem afetar seus requisitos de confiabilidade e manutenção, especialmente quando combinados com dados de outros locais da própria infraestrutura ou de fora do sistema de água. (Como dados meteorológicos).

Sistemas de análise de dados poderiam fazer exatamente isso. Às vezes, simplesmente armazenando e visualizando os dados de maneira amigável ao usuário, por exemplo, em um mapa, podem ser obtidas grandes informações. A análise de dados provenientes de ambientes de Big Data, poderia adicionar modelos preditivos para finalmente olhar para o futuro, possibilitando a manutenção no momento certo e local de forma a otimizar ainda mais o equilíbrio entre desempenho e confiabilidade.

Estas análises também podem evitar desastres causados pelo homem, como quedas súbitas na qualidade da água ou o surto de uma doença contagiosa [2].

http: / / dx.doi.org/10.25286/repa.v3i3.937 
Neste artigo, propomos algumas analises e ações que já podem ser tomadas, mesmo com poucos dados disponíveis, não nos limitando apenas a visualização dos dados gerados.

\section{Gestão de Águas Subterrâneas}

\subsection{Importância da Gestão dos Poços}

Um dos parâmetros mais importantes para monitorar nestas águas é a condutividade, pois os altos níveis de condutividade indicam a salinização das águas subterrâneas e a impossibilidade para consumo humano.

A contaminação das águas subterrâneas geralmente está ligada ao escoamento e infiltração de águas superficiais das áreas urbanas e agrícolas. O problema da salinização está relacionado às zonas costeiras onde o aquífero chega perto do mar. Às vezes, é muito difícil evitar misturar água doce com água do mar. Isso ocorre porque a água doce emerge em algumas áreas marinhas, mas se muita água é extraída do aquífero, então, a água do mar pode infiltrar-se para o aquífero. Se isso acontecer, a água subterrânea não será potável por um longo período de tempo. Além disso, as mudanças climáticas também influenciam o agravamento desse efeito.

O processo de urbanização também afeta o equilíbrio natural devido à impermeabilização do solo que reduz a infiltração local. De acordo com trabalhos publicados por Padowski et al. [3] dentre as 70 maiores cidades do mundo, 28 delas (40\%) usam apenas fontes de água subterrânea. Destas 28 cidades, apenas seis deles usam um reservatório não ameaçado, 20 dependem de um reservatório ameaçado e dois em um vulnerável. No entanto, as simulações para 2.040 mostram que apenas três dessas cidades terão seus reservatórios ainda não ameaçados e cinco cidades terão reservatórios vulneráveis. Os aquíferos estão em perigo e esses aquíferos são a fonte de água de muitas pessoas.

Nas últimas décadas, a população está aumentando e o efeito da migração é que essa população se concentra nas áreas costeiras, de modo que as cidades das áreas costeiras sofreram um grande crescimento em poucos anos. Usando dados das cidades com mais de 1 milhão de habitantes [4], foi calculado a porcentagem de cidades que podem ser consideradas cidades costeiras (50 km ou mais perto do mar). A distribuição das cidades com mais de 1 milhão de habitantes é mostrada na Figura 1 e essas cidades são representadas em diferentes cores, dependendo da proximidade com o mar. Pode-se ver pela Figura 1 que a maioria das grandes cidades estão localizadas próximas a costa.

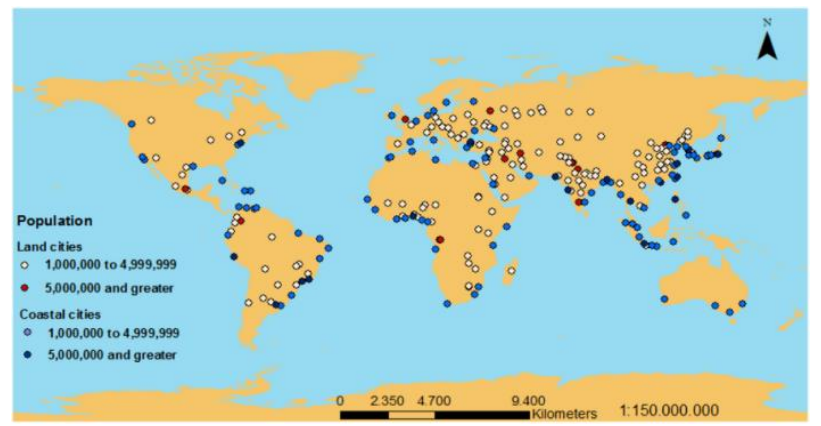

Figura 1 - Distribuição de cidades com mais de 1 milhão de habitantes [4]

As águas subterrâneas são o abastecimento de água para uma grande parte da população mundial. A maior parte da população mundial vive em zonas costeiras. Nessas áreas, a água subterrânea é suscetível a intrusão salina, o que pode tornar a água inalterável. O processo de urbanização, as mudanças climáticas e o bombeamento das águas subterrâneas podem alterar o equilíbrio do aquífero costeiro entre o sal e a água doce. Todos esses fatos levam a uma situação alarmante para a sustentabilidade das cidades e alguns pesquisadores já mencionaram e racionalizaram a necessidade do monitoramento das águas subterrâneas [5,6], enquanto outros estão fazendo algumas medidas e simulações.

No entanto, estas simulações não são tão precisas devido ao alto número de variáveis que afetam cada aquífero. Cada cidade deve ser considerada como um caso específico que requer monitoramento e diagnóstico específicos [6]. Atualmente para monitorar os parâmetros das águas subterrâneas e, especificamente, a salinidade, os esforços são baseados na amostragem manual de poços com periodicidade temporal diferente. Esta metodologia acarreta um desperdício de energia, dinheiro e esforços 
humanos em comparação com a possibilidade de usar dispositivos IoT. O uso destes dispositivos para monitoramento de aquíferos possibilita a coleta de dados em tempo real e é uma boa opção para o monitoramento de longo prazo.

\subsection{Problema de Água Subterrânea em Recife}

O grave problema no fornecimento de água em Pernambuco tem obrigado a população a recorrer à perfuração de poços. A Região Metropolitana do Recife possui mais de 5.000 poços reconhecidos atualmente, que precisam ser monitorados. Um exemplo clássico é a exploração de águas subterrâneas que aconteceu no bairro de Boa Viagem a partir da estiagem rigorosa entre 1997 e 1998. Na ocasião, houve um aumento de novas perfurações ocasionando o rebaixamento do nível piezométrico, que ocorre quando se retira mais do que o aquífero é capaz de renovar e suas recargas naturais se tornam insuficientes, e a salinização local constatada, que ocorre com poços mal perfurados no litoral, quando a água do mar encontra a água doce, por causa do desequilíbrio na pressão do subsolo, em algumas unidades de captação [7].

Poços artesianos, desde que planejados e executados corretamente, podem resolver parte do problema, mas precisam de controle rigoroso do volume de água extraído através de hidrômetros. Desde então, os novos poços recebem uma outorga que lhes permite extrair um volume diário limitado de água do solo.

O problema, portanto, está na fiscalização dos poços, dos volumes extraídos e do nível de condutividade de cada um. As respostas das questões abaixo ajudam no monitoramento e na tomada de decisões importantes para esse controle e fiscalização:

- Quais os volumes de água extraídos dos poços?

- Quais os picos de consumo por período?

- Quais os níveis de condutividade elétrica dos reservatórios aquíferos por bairros da RMR?

As respostas para essas perguntas podem ser obtidas através da análise de dados. Com os dados fornecidos pelos hidrômetros instalados e equipamentos que possam medir o nível de condutividade é possível relacionar com as informações de horários e locais onde estão instalados e extrair informações importantes para o controle de consumo por poço ou por região e verificar se um aquífero está próximo do seu limite de consumo, possibilitando aos órgãos reguladores a tomada de ações com o objetivo de evitar o desgaste destes reservatórios e possíveis problemas ambientais.

\section{Solução IoT para Gestão dos Recursos de Água Subterrânea}

\subsection{Trabalhos Relacionados}

Em 2007 foi lançado, no condado de Rocky View, em Alberta, Canadá, um programa chamado Rocky View Well Watch [8]. Um dos principais objetivos deste projeto foi formar uma rede comunitária de monitoramento de águas subterrâneas usando informações voluntárias dos donos dos poços. Os dados utilizados no projeto eram do nível da agua e a coleta era feita manualmente e de forma voluntária pelos proprietários dos poços.

A fim de fazer previsões sobre como as mudanças nas práticas de uso da terra e clima afetaram o balanço hídrico no condado de Rocky View, os pesquisadores utilizaram os dados obtidos para criar modelos numéricos para simular possíveis resultados. Variáveis que afetam a elevação do nível da água foram utilizadas nestes modelos como, clima, recarga de água subterrânea, geologia, e bombeamento de águas subterrâneas. No site do projeto é possível verificar um mapa com os poços e os níveis de agua de cada um deles.

Muitas pesquisas ainda estão sendo feitas na área do uso de IoT para gestão de águas. Alguns sistemas que já fazem uso dessa tecnologia têm provado que uma redução da intervenção humana pode alcançar um aumento significativo de eficiência.

Monitoramento e gerenciamento contínuo do meio ambiente através da integração do IoT e sistemas que utilizem sensores é um tópico ativo para pesquisadores, engenheiros e até

http://dx.doi.org/10.25286/repa.v3i3.937 
administradores públicos. Como trabalhos notáveis nessa área de sistemas de gestão de água, podemos citar o Sustainable Water Distribution Strategy with Smart Water Grid [9], que explica como preencher a lacuna entre conectar vários recursos hídricos e otimizar a gestão do sistema com novas soluções de tecnologia de informação. Água da chuva, recursos externos, e outros tipos alternativos de recursos hídricos são integrados nesse sistema para fornecer um ambiente sustentável.

$O$ artigo intitulado Internet of Things for Smart cities [10] fornece uma visão sobre a realização de uma rede IoT junto com a rede e os serviços de back-end necessários. O artigo também descreve os protocolos utilizados nesta arquitetura.

O artigo Micro Water distribution resources [11] descreve sobre a construção sustentável de um sistema de distribuição de água em vilas na Índia, considerando fatores locais como trabalho, necessidades da comunidade, clima e tempo de implementação. $O$ artigo dá uma ideia sobre os vários desafios que tiveram que enfrentar ao construir esse sistema na Índia.

\subsection{Projeto de Sustentabilidade Hídrica do Governo do Estado de Pernambuco}

Atualmente o controle do volume de consumo por poço é feito manualmente, onde cada proprietário do poço anota a informação de consumo e envia mensalmente a Agência Pernambucana de Águas e Clima - APAC. Esse fluxo de informação demanda bastante tempo para ser concluído, o que acarreta em uma demora na análise destes dados e de um completo mapeamento dos índices de consumo de toda a região, impactando ainda na tomada de possíveis ações de controle. As informações sobre os índices de condutividade são inexistentes.

Pensando na situação atual e em uma possível solução, o Governo do Estado desenvolveu um projeto chamado de Projeto de Sustentabilidade Hídrica de Pernambuco - PSHPE, que visa a instalação e monitoramento, através da Agência Pernambucana de Águas e Clima - APAC, de 356 poços artesianos na Região Metropolitana do Recife.
Esta solução para gestão, otimização, monitoramento e controle dos sistemas de água subterrânea, consiste na implantação de uma rede de monitoramento dos poços através de sistema de telemetria (IoT), em poços da Região Metropolitana do Recife. Nesta rede, serão instalados hidrômetros, módulos de comunicação remota, que permitem a comunicação com um sistema informatizado, onde se torna possível conferir as leituras (vazões) dos hidrômetros, e sondas que permitem o monitoramento do nível e da salinidade da água destes poços. Esta rede de monitoramento requer uma continuidade na coleta de dados, tendo como objetivo a obtenção de um monitoramento adequado para os aquíferos.

Este projeto prevê a instalação dos seguintes itens, de acordo a Figura 2:

(a) Módulos de comunicação remota: Equipamento responsável pela promoção da conexão lógica dos parâmetros monitorados nos poços com o servidor de banco de dados remoto.

(b) Hidrômetros de telemetria: Equipamento responsável pelo registro do consumo de agua com conexão do tipo Reed switch.

(c) Sistema de monitoramento: Software para análise e controle do sistema de monitoramento instalado.

(d) Sondas para monitoramento de nível d'água e de salinidade da água: Os Sensores de medição de nível d'água são do tipo cerâmicocapacitivo para envio em tempo real de informações sobre o nível de coluna de água acima da posição em que o sensor estiver instalado no poço, suportam operação a uma profundidade de até $100 \mathrm{~m}$ de coluna de água e possui acessórios que permitam sua instalação a até $200 \mathrm{~m}$ do nível do solo, operam na faixa de $1 \mathrm{a}$ $1.000 \mu \mathrm{S}$ (micro-siemens). 


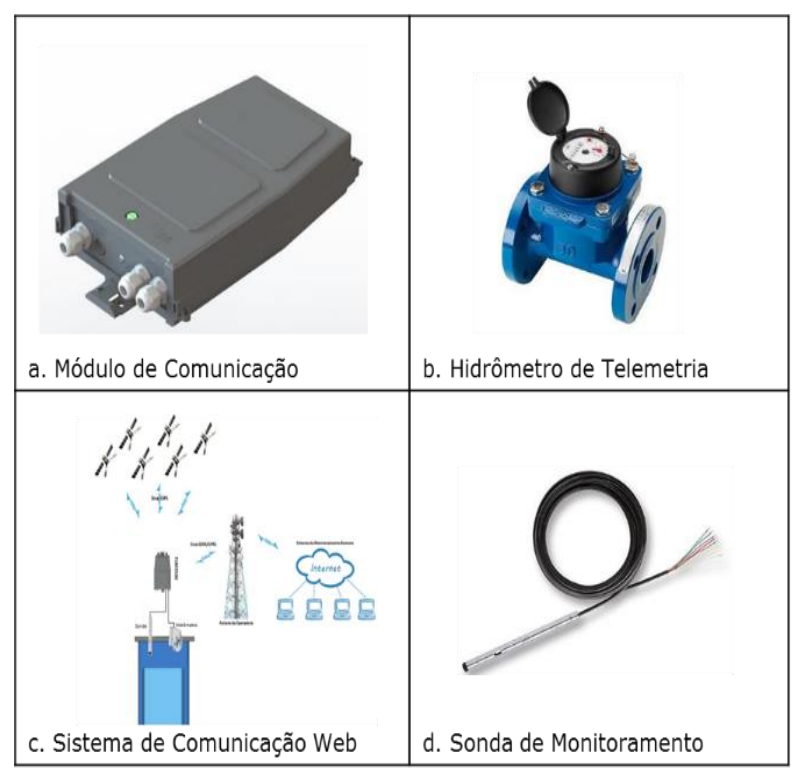
IoT

Figura 2 - Equipamentos que compõem a solução

\section{Análises Propostas com os Dados Obtidos}

\subsection{Análise Preliminar}

A solução proposta pelo Projeto de Sustentabilidade Hídrica de Pernambuco - PSHPE otimiza e automatiza o envio das informações para o órgão regulador, APAC. Com os dados em tempo real, é possível visualizar o que acontece e o que pode ser feito.

Com a aplicação de técnicas de análise de dados, podemos gerar gráficos de consumo, verificar os níveis de condutividade e identificar quais poços e regiões já possuem um esgotamento no aquífero, e com o passar do tempo, de posse de dados históricos gerados por este sistema, prever quais aquíferos serão impactados, quais regiões vão ter aumento de consumo e desta forma, tomar ações preditivas.

De posse dos dados provenientes de 20 módulos de comunicação, com hidrômetro de telemetria e sonda de condutividade, podemos iniciar algumas análises.

Os módulos estão instalados pela região metropolitana do Recife, de acordo a Figura 3, e enviam informações de consumo, condutividade e temperatura dos poços a cada cinco minutos.
Os dados obtidos correspondem ao período de 15 de julho de 2017 a 6 de dezembro de 2017.

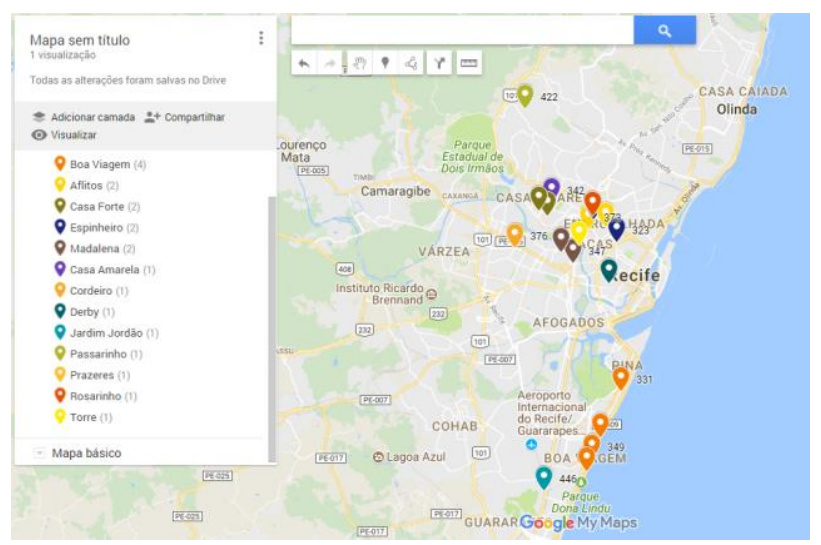

Figura 3 - Módulos instalados pela Região Metropolitana de Recife

Como no início nem todos os poços analisados estavam conectados ao sistema, então, ao longo do período fomos obtendo mais informações de mais poços.

A Figura 4, mostra um exemplo de como são as colunas dos dados como foram obtidos:

\begin{tabular}{|c|c|c|c|c|c|c|c|c|}
\hline 4 & A & B & $c$ & $D$ & $F$ & G & H & I \\
\hline 1 & $\operatorname{cod}$ & cod modulo & volume & dh leitura & contad icm & nivel poco & temperatura & analog1 \\
\hline 2 & 7541776 & 323 & 0.000 & $04 / 07 / 201717: 45$ & $O N ; N$ & $\mathrm{~N} ; \mathrm{N}$ & $\mathrm{N} ; \mathrm{N}$ & $\mathrm{N} ; 4.183^{\prime \prime}$ \\
\hline 3 & 7566757 & 342 & 0.000 & $12 / 07 / 201712: 59$ & $O \mathrm{~N} ; \mathrm{N}$ & $\mathrm{N} ; \mathrm{N}$ & $\mathrm{N} ; \mathrm{N}$ & $N ; 4,146^{\prime \prime}$ \\
\hline 4 & 7566771 & 342 & 0.000 & $12 / 07 / 201712: 54$ & $1 \mathrm{~N} ; \mathrm{N}$ & $\mathrm{N} ; \mathrm{N}$ & $\mathrm{N} ; \mathrm{N}$ & $N ; 4,151^{\prime \prime}$ \\
\hline 5 & 7566785 & 342 & 0.000 & $12 / 07 / 201712: 59$ & $2 \mathrm{~N} ; \mathrm{N}$ & $\mathrm{N} ; \mathrm{N}$ & $\mathrm{N} ; \mathrm{N}$ & $N ; 4.151^{\prime \prime}$ \\
\hline 6 & 7566798 & 342 & 0.000 & $12 / 07 / 201713: 04$ & $3 \mathrm{~N} ; \mathrm{N}$ & $\mathrm{N} ; \mathrm{N}$ & $\mathrm{N} ; \mathrm{N}$ & $N ; 4.151^{\prime \prime}$ \\
\hline 7 & 7566814 & 342 & 0.000 & 12/07/201713:09 & $4 \mathrm{~N} ; \mathrm{N}$ & $\mathrm{N} ; \mathrm{N}$ & $\mathrm{N} ; \mathrm{N}$ & $\mathrm{N} ; 4,150^{\prime \prime}$ \\
\hline 8 & 7566827 & 342 & 0.000 & 12/07/2017 13:14 & $5 \mathrm{~N} ; \mathrm{N}$ & $\mathrm{N} ; \mathrm{N}$ & $\mathrm{N} ; \mathrm{N}$ & $N ; 4.151^{\prime \prime}$ \\
\hline 9 & 7566841 & 342 & 0.000 & $12 / 07 / 201713: 19$ & $6 \mathrm{~N} ; \mathrm{N}$ & $\mathrm{N} ; \mathrm{N}$ & $\mathrm{N} ; \mathrm{N}$ & $\mathrm{N} ; 4,150^{\prime \prime}$ \\
\hline 10 & 7566855 & 342 & 0.000 & $12 / 07 / 201713: 24$ & $7 \mathrm{~N} ; \mathrm{N}$ & $\mathrm{N} ; \mathrm{N}$ & $\mathrm{N} ; \mathrm{N}$ & $\mathrm{N} ; 4.150^{\prime \prime}$ \\
\hline 11 & 7566869 & 342 & 0.000 & $12 / 07 / 201713: 29$ & $8 \mathrm{~N} ; \mathrm{N}$ & $\mathrm{N} ; \mathrm{N}$ & $\mathrm{N} ; \mathrm{N}$ & $N ; 4.149 "$ \\
\hline 12 & 7566883 & 342 & 0.000 & $12 / 07 / 201713: 34$ & $9 \mathrm{~N} ; \mathrm{N}$ & $\mathrm{N} ; \mathrm{N}$ & $\mathrm{N} ; \mathrm{N}$ & $N ; 4.151^{\prime \prime}$ \\
\hline 13 & 7566897 & 342 & 0.000 & 12/07/2017 13:39 & $10 \mathrm{~N} ; \mathrm{N}$ & $\mathrm{N} ; \mathrm{N}$ & $\mathrm{N} ; \mathrm{N}$ & $N ; 4.150^{\prime \prime}$ \\
\hline
\end{tabular}

Figura 4 - Exemplo da coluna da tabela dos dados obtidos

\subsection{Pré-Processamento}

Os dados estavam no formato bruto e foram necessários procedimentos de limpeza e organização dos dados.

Foi incluída uma coluna período, cujos valores correspondem aos turnos Madrugada, Manhã, Tarde e Noite, onde:

http://dx.doi.org/10.25286/repa.v3i3.937 
Horário das 5:00 as $11: 59$, corresponde ao turno Manhã;

Horário das $12: 00$ as $17: 59$, corresponde ao turno Tarde;

Horário da $18: 00$ as 23:59, corresponde ao turno Noite;

Horário das $00: 00$ as 4:59, corresponde ao turno Madrugada;

Foi incluída também uma coluna com a informação do Bairro onde o modulo está instalado.

Com os dados limpos e organizados, foi possível a geração de gráficos, de acordo a Figura 5 , com o intuito de obter algumas informações preliminares.

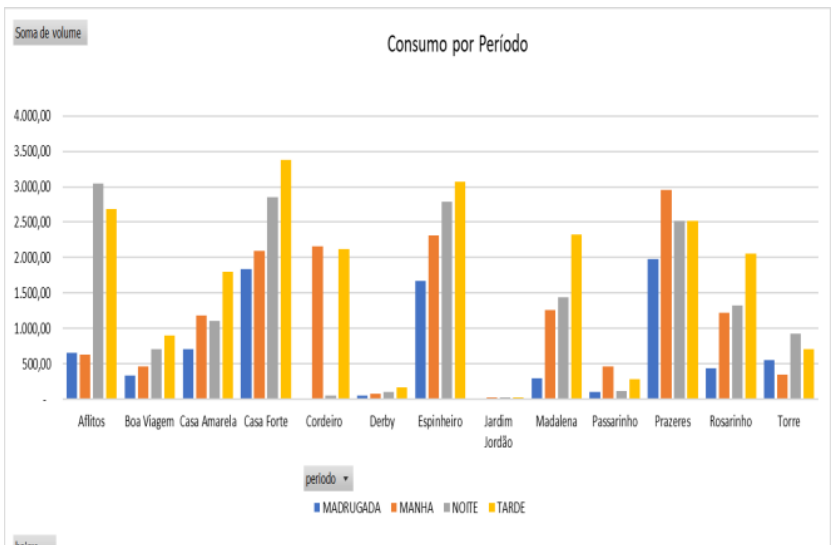

Figura 5 - Gráfico de Consumo por Período

Tabela 1 - Consumo por Bairro e Período Volume por Periodo Rótulos de Colunk:

\begin{tabular}{|c|c|c|c|c|c|}
\hline ulos & - MADRUGADA & MANHA & NOTE & TAFEE & Total G \\
\hline Aflitos & 607,30 & 635,10 & 30099,50 & 2688,00 & $7.019,90$ \\
\hline Boa Viagem & 333,20 & 462,40 & 705,80 & 903,90 & 24053 \\
\hline Casa Amarela & 714,40 & $1.189,70$ & $1.100,10$ & $1.794,50$ & $4.798,70$ \\
\hline Casa Forte & $1.843,30$ & 2100,80 & 2851,30 & $3.378, \infty 0$ & $10.174, \infty 0$ \\
\hline Cordeiro & & 2161,40 & 52,60 & $2118, \infty 0$ & $4,332,60$ \\
\hline roy & 53,30 & $76, \infty$ & 106,80 & 173,70 & 409,80 \\
\hline Espinheir & $1.675, \infty 0$ & 2313,10 & 2782,80 & $3.070,00$ & $9.841,50$ \\
\hline Jardim Jordã̃ & 15,70 & 28,30 & 29,90 & 2,30 & 97,20 \\
\hline Madalena & 207,70 & $1.255,70$ & $1.435,10$ & 2325,10 & $5,313,00$ \\
\hline Passarinho & 101,30 & 459,20 & 117,40 & 200,10 & 968,00 \\
\hline Prazeres & $1.981,10$ & 2950,10 & 2525,30 & 2515,40 & $9.971,90$ \\
\hline osarinho & 438,40 & $1.218,80$ & $1,320,30$ & 2061,80 & $5.009,30$ \\
\hline ime & 507,90 & 346,50 & 928,30 & $711, \infty$ & 2543,70 \\
\hline tal Gerc & $8.669,20$ & $15.197,10$ & $16.995,20$ & 2054,00 & 62915,50 \\
\hline
\end{tabular}

Pela informação do gráfico, na Figura 5, e da Tabela 1, podemos concluir que o período de maior consumo de água dos poços artesianos, em metros cúbicos, é no período da tarde, seguido do período da noite e manha, sendo o menor consumo no período da madrugada.

Pode-se constatar também que o bairro que tem o maior consumo pelo período da manhã é Prazeres, pelo período da tarde é Espinheiro e pelo período da noite é Aflitos.

\subsection{Análise de Condutividade}

A condutividade elétrica da água representa a facilidade ou dificuldade de passagem da eletricidade na água. A Tabela 2 apresenta a condutividade por bairro e por módulo.

Tabela 2 - Condutividade Por Bairro e Módulo.

\begin{tabular}{|c|c|c|}
\hline Bairros & Média de icm & DesvPad de icm \\
\hline \multicolumn{3}{|l|}{ Aflitos } \\
\hline 373 & 0,37 & 0,02 \\
\hline 386 & 0,12 & 0,00 \\
\hline \multicolumn{3}{|c|}{ Boa Viagem } \\
\hline 331 & $1.739,59$ & 613,39 \\
\hline 349 & 0,29 & 0,06 \\
\hline 388 & $6.517,29$ & $2.297,34$ \\
\hline 395 & 0,25 & 0,01 \\
\hline \multicolumn{3}{|c|}{ Casa Amarela } \\
\hline 342 & 0,13 & 0,01 \\
\hline \multicolumn{3}{|c|}{ Casa Forte } \\
\hline 346 & 0,40 & 0,03 \\
\hline 378 & 0,61 & 0,00 \\
\hline \multicolumn{3}{|c|}{ Cordeiro } \\
\hline 376 & 0,75 & 0,01 \\
\hline \multicolumn{3}{|l|}{ Derby } \\
\hline 387 & $2.486,47$ & $1.275,72$ \\
\hline \multicolumn{3}{|c|}{ Espinheiro } \\
\hline 323 & 0,35 & 0,01 \\
\hline 377 & 0,19 & 0,02 \\
\hline \multicolumn{3}{|c|}{ Jardim Jordão } \\
\hline 446 & 0,14 & 0,01 \\
\hline \multicolumn{3}{|c|}{ Madalena } \\
\hline 347 & 0,64 & 0,02 \\
\hline 365 & 164,73 & 382,67 \\
\hline \multicolumn{3}{|c|}{ Passarinho } \\
\hline 422 & 0,07 & 0,00 \\
\hline \multicolumn{3}{|c|}{ Prazeres } \\
\hline 366 & $1.246,33$ & 486,19 \\
\hline \multicolumn{3}{|c|}{ Rosarinho } \\
\hline 411 & 0,17 & 0,01 \\
\hline \multicolumn{3}{|l|}{ Torre } \\
\hline 394 & 0,88 & 0,02 \\
\hline Total G & 253,63 & 658,57 \\
\hline
\end{tabular}

De acordo a Tabela 2, é possível verificar que alguns módulos instalados no Bairro de Boa Viagem, possuem altos índices de condutividade, 
que indicam que a água é salina. Em alguns pontos como Prazeres e Derby, a água está como a de um Rio marginal e salobra. Para o caso estudado, os bairros que obtiveram altas médias de índices de condutividade, são as que possuem altos valores de desvio padrão, o que indica que a condutividade nesses locais varia bastante, podendo estar relacionadas a fatores como aumento no consumo, ou variação nos índices de chuva no local. Nas Figuras 6, 7 e 8, são listadas as localizações em que estes módulos.

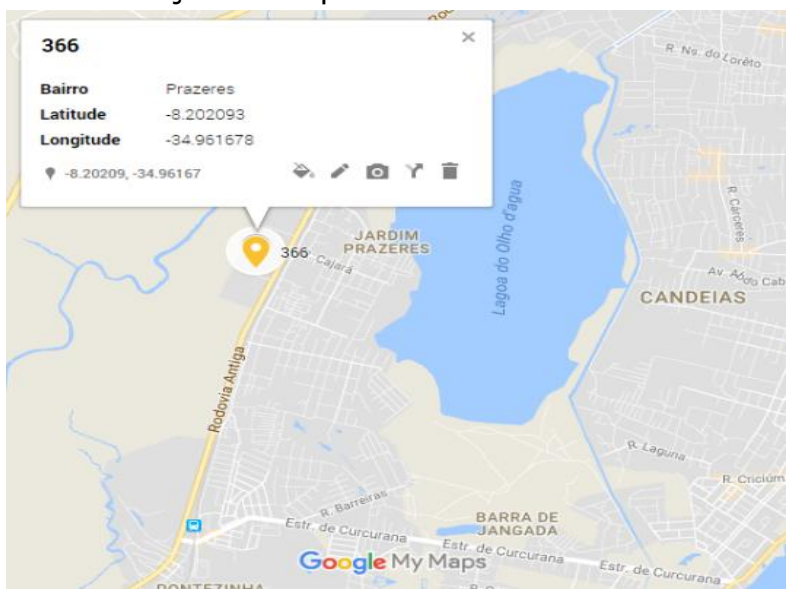

Figura 6 - Módulo instalado no bairro de Prazeres, próximo a lagoa do Olho d'agua

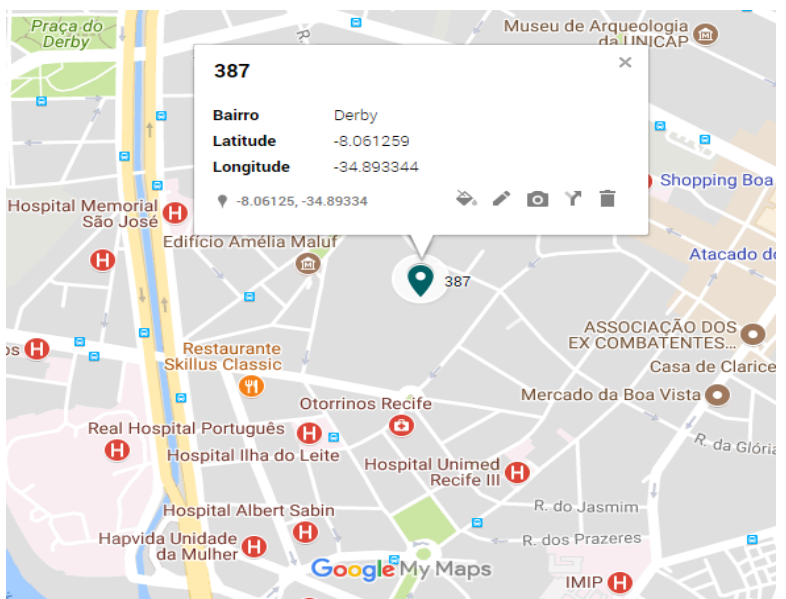

Figura 7 - Módulo instalado no bairro do Derby, próximo ao canal

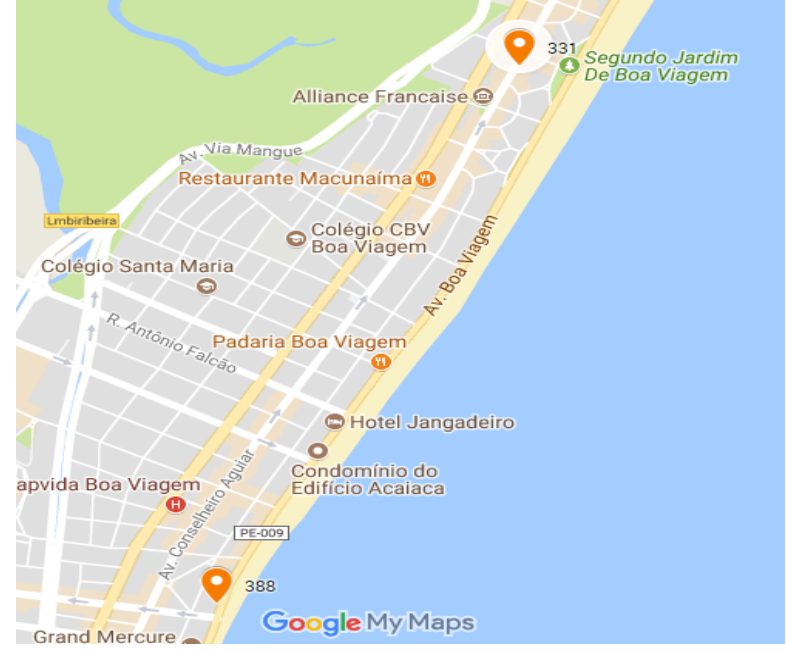

Figura 8 - Módulos instalados no bairro de Boa Viagem, próximos ao mar.

Podemos constatar que os módulos estão em poços localizados próximo a um lago, ao canal, e próximo a beira mar, o que pode ser um indicio do alto valor da condutividade encontrado nos poços destes locais.

Com essas análises preliminares podemos destacar os seguintes pontos:

Na Região Metropolitana de Recife, o consumo desenfreado que aconteceu, a partir da estiagem rigorosa que aconteceu entre 1997 e 1998, no bairro de Boa Viagem, ocasionou um rebaixamento do nível piezométrico, ocasionando um aumento na salinidade da água $e$ consequentemente, aumento da condutividade. Fato este que podemos comprovar através das análises preliminares obtidas, onde verificamos que em dois módulos instalados no bairro de Boa Viagem, os índices de condutividades mostram que a água é salina.

\subsection{Correlação entre Índice de Chuva, Maré e Condutividade}

É possível encontrar alguns trabalhos que tentam correlacionar a influência dos índices de chuvas com o nível dos poços [12]. Como ainda não possuímos dados concretos sobre os níveis de poços da região, vamos verificar se existe alguma correlação entre os índices de maré e chuva com a condutividade dos poços. Para isso, foi obtido no site da APAC [13] os dados históricos de chuva de todo o estado de Pernambuco. No site Tábua 
de Marés [14] também foi possível obter o nível das marés do estado.

De posse desses dados, selecionados o período que compreende entre os meses de setembro a novembro de 2017 e os poços que possuem dados completos desse período. Realizamos a limpeza dos dados e tentamos correlacionar com os dados dos poços disponíveis. Distribuímos na Tabela 4, os dados de condutividade, índices de chuva e maré, separados por período e por poço.

Tabela 4 - Valores de condutividade, nível mare e índice de chuvas por poço, dia e período.

\begin{tabular}{|c|c|c|c|}
\hline Rótulos de Linha & Média de icm & Média de nivel_mare & Média de indice_chuvas \\
\hline$\Xi 323$ & 0,35 & 1,32 & 0,78 \\
\hline$\Xi_{\text {set }}$ & 0,35 & 1,31 & 1,61 \\
\hline \multicolumn{4}{|l|}{$\boxminus 01 /$ set } \\
\hline MADRUGADA & 0,35 & 0,50 & - \\
\hline MANHA & 0,35 & 2,10 & - \\
\hline NOITE & 0,35 & 1,90 & - \\
\hline TARDE & 0,35 & 0,60 & - \\
\hline \multicolumn{4}{|l|}{$\boxminus 02 /$ set } \\
\hline MADRUGADA & 0,35 & 0,60 & - \\
\hline
\end{tabular}

Como estamos tentando correlacionar duas variáveis de escala métrica, o coeficiente de correlação de Pearson, ou $r$ de Pearson, é o mais indicado. $\mathrm{O} r$ de Pearson pode ser obtido através da fórmula (1) abaixo:

$$
r=\frac{\sum(x i-\underline{x})(y i-\underline{y})}{\sqrt{\left(\sum \quad(x i-\underline{x}) 2\right)\left(\sum(y i-\underline{y})^{2}\right)}}
$$

Onde $x_{i}$ e $y_{\square} s a ̃ o$ os valores das variáveis que vamos correlacionar, $\underline{x}$ e $\underline{y}$ são respectivamente as médias dos valores de $x_{\square}$ e $y_{\square}$.

De posse da Tabela 4, aplicamos a formula de correlação $r$ de Pearson para verificar a correlação existente entre esses dados, para cada poço, como mostrado na Tabela 5.

Tabela 5 - Correlação entre condutividade, nível mare e índice de chuvas por poço.

\begin{tabular}{|r|r|r|r|r|r|}
\hline Poço & icm & nivel_mare indice_chuvas & $\begin{array}{c}\text { Correlação icm } \mathbf{x} \\
\text { nivel_mare }\end{array}$ & $\begin{array}{c}\text { Correlação icm } \mathbf{x} \\
\text { indice_chuvas }\end{array}$ \\
\hline 323 & 0,35 & 1,32 & 0,78 & 0,05 & 0,16 \\
\hline 342 & 0,13 & 1,37 & 0,82 & 0,02 & 0,07 \\
\hline 346 & 0,39 & 1,38 & 0,86 & 0,05 & 0,23 \\
\hline 349 & 0,29 & 1,24 & 1,19 & 0,00 & 0,04 \\
\hline 365 & 172,35 & 1,32 & $0,89-$ & 0,11 & 0,11 \\
\hline 366 & $1.218,00$ & 1,29 & $0,94-$ & 0,03 & 0,14 \\
\hline 373 & 0,37 & 1,23 & 1,15 & 0,05 & 0,12 \\
\hline 376 & 0,75 & 1,35 & 0,88 & $0,01-$ & 0,32 \\
\hline 378 & 0,61 & 1,33 & 0,94 & 0,02 & - \\
\hline 386 & 0,12 & 1,33 & 0,93 & 0,05 & - \\
\hline
\end{tabular}

Pela Tabela 5, os índices de correlação, para todos os poços, estão próximos de zero, o que indica que não há relação entre as variáveis de condutividade, nível de maré e índice de chuvas. Geramos também o gráfico de dispersão para cada poço.

Nas Figuras 9 e 10 é mostrado apenas, como exemplo, o gráfico de dispersão correspondente ao poço 323.

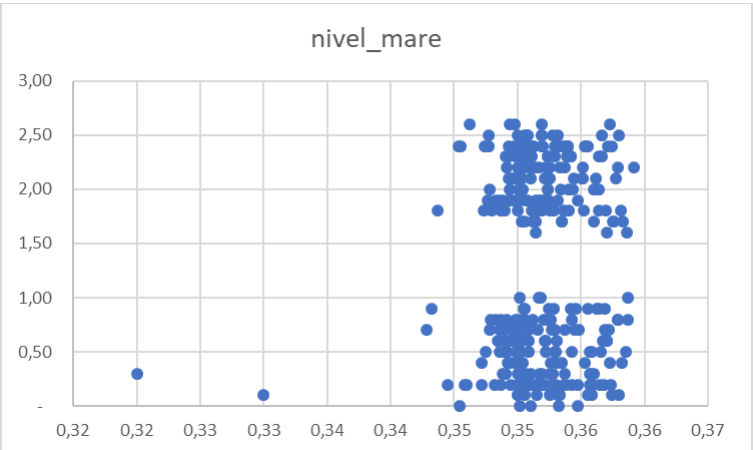

Figura 9 - Gráfico de dispersão do nível mare com a condutividade do poço 323.

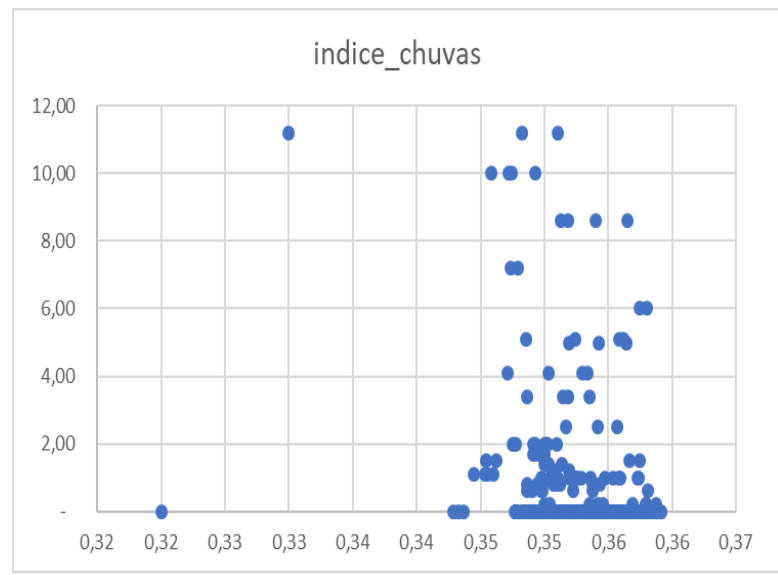

Figura 10 - Gráfico de dispersão do índice de chuvas com a condutividade do poço 323

Pelas Figuras 9 e 10, é possível observar que não existe uma relação entre as variáveis condutividade, índice de chuva e nível maré. Os demais poços possuem gráficos semelhantes.

\section{Análises e Discussões}

Importante destacar que para se obter um melhor cenário, mais dados devem ser obtidos. 
Estamos apenas avaliando um período de amostragem de 3 meses e de apenas 20 poços que já possuem o sistema de telemetria instalado.

Com relação as análises de índices de consumo por mês, mais dados são necessários, assim como um maior período de amostragem para que possamos elaborar uma análise mais detalhada.

Com as análises preliminares podemos destacar alguns pontos:

No bairro de Boa Viagem, na Região Metropolitana de Recife, é possível comprovar através das análises preliminares obtidas, que em dois módulos instalados os índices de condutividades mostram que a água é salina, fato este que pode constatar um rebaixamento no nível piezométrico do aquífero na região.

O consumo por período nos permite gerar um perfil de consumo dos poços. Com a análise feita, constatamos que o período da tarde apresenta o maior consumo, fato este que pode estar relacionado ao período de retorno do trabalho e/ou escola.

\section{Conclusão}

Apesar de abundantes, as águas subterrâneas não são inesgotáveis, e o seu uso sem uma gestão consciente pode trazer problemas a curto e a médio prazo.

A extração de água subterrânea de forma exagerada, que ultrapassa os limites de produção das reservas reguladoras ou ativas do aquífero, ocasiona um processo de rebaixamento do nível do aquífero e irá provocar danos ao meio ambiente ou para o próprio recurso. Portanto, a água subterrânea pode ser retirada de forma permanente e em volumes constantes, por muitos anos, desde que esteja condicionada a estudos prévios do volume armazenado no subsolo e das condições climáticas e geológicas de reposição[15].

Para a correta gestão do uso de águas subterrâneas é necessário um monitoramento continuo dos parâmetros de consumo e condutividade. Os índices de condutividade são um parâmetro crucial para determinar se um aquífero está sendo prejudicado. A dificuldade de um monitoramento continuo pode ser resolvida com o uso de soluções IoT que possa proporcionar, em tempo real, a informação desses parâmetros [16].

Destacamos neste artigo a importância das águas subterrâneas e de sua gestão, assim como a implantação de uma solução do Governo do Estado para controle no uso dessas águas.

A Solução IoT, ainda em fase de implantação pelo Governo do Estado, se mostra eficaz no envio de informações em tempo real do que acontece em cada poço, porém não possibilita uma análise completa de todo o cenário.

Propomos neste artigo, uma análise dos dados gerados, em conjunto, de todos os poços, com o uso dos dados provenientes da Solução IoT. Geramos gráficos de análise do consumo por bairros e por período, o que nos permite visualizar qual o período do dia que o consumo é maior. Geramos também uma análise dos níveis de condutividade da Região.

Com mais dados disponíveis, melhores análises poderão ser efetuadas, e com o uso de dados históricos e aplicação de técnicas de aprendizagem de máquina, com a seleção adequada de atributos, como foi efetuado neste artigo para as analises, podemos gerar previsões sobre quais bairros terão um aumento de consumo e de índice de condutividade, possibilitando a tomada de ações preditivas afim de evitar o esgotamento dos aquíferos e impactos ambientais significativos.

\subsection{Trabalhos Futuros}

O monitoramento em tempo real pode fornecer insights ao vivo sobre o estado corrente da rede de monitoramento de águas subterrâneas. Com a solução IoT implantada, os dados de consumo, nível e condutividade da água podem ser obtidos em tempo real. Isso possibilita detectar estados críticos e acionar uma ação de resposta. No sistema IoT do projeto de sustentabilidade hídrica, cada poço possui sensores de temperatura, nível, condutividade e consumo. Os dados são enviados a cada 5 minutos. Quando o projeto estiver finalizado, teremos dados de 100 poços, enviando 4 medidas a cada 5 minutos, num total de 4.800 medições por hora.

http://dx.doi.org/10.25286/repa.v3i3.937 
A análise dos dados de consumo de água, nível, condutividade e temperatura é caracterizada por atualizações incrementais ao longo do tempo. Para essa configuração, o processamento de fluxo e a tecnologia CEP podem ser uma solução. Para deduzir o estado atual de um sistema, é necessário apenas cálculos sobre as últimas informações do sensor. Portanto, o estado que é necessário para processamento é relativamente pequeno. $O$ processamento CEP, mantêm o estado na memória e, assim, permitem um alto rendimento. O Uso do mecanismo CEP de código aberto poderia executar as análises necessárias para os 100 poços em paralelo em uma única máquina. O desempenho vai depender dos detalhes de implementação e das análises solicitadas.

\section{Referências}

[1] STAMFORD. Gartner Says 6.4 Billion Connected "Things" Will Be in Use in 2016, Up 30 Percent From 2015. Newsroom,10 Nov. 2015.

Disponível em:

<https://www.gartner.com/newsroom/id/316531 7>. Acesso em: 2 mar. 2018.

[2] CHEUNG, Cy; NUIJTEN, Martijn. Big Data and the Future of Water Management. Rijksdienst voor Ondernemend Nederland. Disponível em: <https://www.rvo.nl/sites/default/files/2014/05/B ig\%20Data\%20and\%20the\%20Future $\% 20$ of\%20 Water\%20Management.pdf $>$. Acesso em: 18 out. 2017.

[3] PADOWSKI, Julie C.; GORELICK, Steven M. Global analysis of urban surface water supply vulnerability. Environmental Research Letters, v. 9, n. 10, p. $104004,2014$.

[4] Intl ESRI Data. Disponível em: <http://www.baruch.cuny.edu/geoportal/data/esr i/esri_intl.htm\#world> Acesso em: 16 out. 2017.

[5] HAYASHI, T. et al. Effects of human activities and urbanization on groundwater environments: An example from the aquifer system of Tokyo and the surrounding area. Science of the total environment, v. 407, n. 9, p. 3165-3172, 2009. Disponível em:

<http://www.ncbi.nlm.nih.gov/pubmed/18823643

> Acesso em: 16 out. 2017.

[6] FOSTER, S. S. D. The interdependence of groundwater and urbanisation in rapidly developing cities. Urban water, v. 3, n. 3, p. 185-192, 2001.

[7] SANTOS, Ivaneide de Oliveira et al. Aqüífero Boa Viagem, uma Discussão de seus Usos versus suas Potencialidades, Recife-PE. Revista

Brasileira de Geografia Física, v. 4, n. 4, p. 848-856, 2012.

[8] UNIVERSITY OF CALGARY. Research Connection. Groundwater Connections connect, connecting research, community \& education, 2013. Disponível em:

<http://groundwaterconnections. weebly.com/rese arch-connections.html> Acesso em: 17 out. 2017.

[9] BYEON, Seongjoon et al. Sustainable water distribution strategy with smart water grid.

Sustainability, v. 7, n. 4, p. 4240-4259, 2015.Disponível em:

<http://www.mdpi.com/2071-

1050/7/4/4240/htm> Acesso em: 2 fev. 2018.

[10] ZANELLA, A et al. Internet of things for smart cities. IEE Internet of Things Journal, $v$. 1, n. 1, p.22-32, Feb. 2014. Disponível em: <http://ieeexplore.iee.org/stamp/stamp.jsp?arn umber $=6740844>$ Acesso em: 2 fev. 2018.

[11] RAMESH, Maneesha Vinodini et al. Micro water distribution networks: A participatory method of sustainable water distribution in rural communities. In: GLOBAL HUMANITARIAN TECHNOLOGY CONFERCE (GHTC), 2016, Seattle. Proceedings... Seattle: IEEE, 2016. p. 797-804.

[12] SILVA, Iara Lina de S.; MOTA, Elison José; OLIVEIRA, Leidiane Leão de. Relação da água da chuva com os poços de abastecimento público do Urumari em Santarém Pará, Brasil. In: CONGRESSO BRASILEIRO DE ÁGUAS SUBTERRÂNEAS, 13., 2014, Belo Horizonte. Proceedings... Belo Horizonte: ABAS, 2014.. 
[13] Turismo no Recife. Disponível em:

<http://www.turismonorecife.com.br/pt-

$\mathrm{br} /$ informacoes-importantes/tabua-de-mares>

Acesso em: 15 mar. 2018.

[14] Agência Pernambucana de Águas e Climas APAC. Disponível em:

<http://www.apac.pe.gov.br/meteorologia/>

Acesso em: 15 mar. 2018.

[15] Associação Brasileira de Águas

Subterrâneas, ABAS. Disponível em:

$<$ http://www.abas.org/educacao.php > Acesso em

2 mar. 2018.

[16] PERLMAN, Howard. Electrical Conductivity and Water. The USGS Water Science School, 2014. Disponível em:

<http://water.usgs.gov/edu/electrical-

conductivity.html> Acesso em: 1 mar. 2018. 